\title{
La médiatisation du burn out dans la presse écrite
}

Le cas du quotidien Le Monde

The burn out's mediatization on the written press: case study about Le Monde

\section{Christine Chevret-Castellani}

\section{CpenEdition}

\section{Journals}

Édition électronique

URL : https://journals.openedition.org/questionsdecommunication/11178

DOI : 10.4000/questionsdecommunication. 11178

ISSN : 2259-8901

\section{Éditeur}

Presses universitaires de Lorraine

Édition imprimée

Date de publication : 1 septembre 2017

Pagination : 335-353

ISBN : 9782814303256

ISSN : 1633-5961

\section{Référence électronique}

Christine Chevret-Castellani, "La médiatisation du burn out dans la presse écrite », Questions de communication [En ligne], 31 | 2017, mis en ligne le 01 septembre 2019, consulté le 26 octobre 2021. URL : http://journals.openedition.org/questionsdecommunication/11178 ; DOI : https://doi.org/ 10.4000/questionsdecommunication.11178 
CHRISTINE CHEVRET-CASTELLANI

Laboratoire des sciences de l'information et de la communication

Université Paris 13

F-93430

christine.chevret@univ-paris13.fr

\section{LA MÉDIATISATION DU BURN OUT DANS LA PRESSE ÉCRITE LE CAS DU QUOTIDIEN LE MONDE}

Résumé. - Les médias français s'intéressent au syndrome d'épuisement professionnel, surtout depuis la vague de suicides dans les grandes entreprises publiques, en 2007. Nous envisageons l'usage de l'expression « burn out 》 dans la presse écrite en partant de l'idée que le lexique fait l'objet d'enjeux socio-politiques. À partir d'une contextualisation de cet usage et pour circonscrire son introduction dans le débat public, nous le situons plus précisément dans les publications du quotidien Le Monde. Nous dégageons différents moments discursifs qui permettent de situer des significations plurielles de cette expression. Après avoir interrogé les caractéristiques de ces différents moments, nous nous demandons s'il est possible d'envisager un figement de « burn out », voire la construction d'une formule, c'est-à-dire la cristallisation d'enjeux socio-politiques dans le discours.

Mots clés. - burn out, traitement médiatique, presse écrite, Le Monde, néo-capitalisme, logique entrepreneuriale, pathologie sociale 
$L$ 'information sur le phénomène de burn out, syndrome d'épuisement fréquemment apparenté à une souffrance au travail', s'est répandue dans l'espace public à partir de la médiatisation des suicides chez Renault et à EDF, en 2007, puis à France Télécom. C'est surtout en 2009 que l'évocation dans les médias de ce phénomène devient particulièrement significative. Comme l'indique Christian Bourion (2010:214), « le nombre de canaux qui répercutent le thème de la souffrance au travail a littéralement explosé. Au cours de la seule année 2009, il se tient presque autant de manifestations sur ce thème que pendant le reste de la décennie ». On se souvient, par ailleurs, combien la reprise des propos du PDG de France Télécom, Didier Lombard, tenus dans Le Monde (« La première urgence c'est d'arriver à stopper le phénomène de contagion, en finir avec cette mode des suicides », Le Monde, 17/09/2009) a eu pour finalité de susciter l'émotion dans l'opinion. La reprise dans les autres médias a eu pour effet une amplification, en particulier au regard de la répétition de l'expression « mode des suicides $»^{2}$. Pour l'analyser, nous partons de l'idée de « moments discursifs 》 (Moirand, 2007) dans le traitement de l'information, d'abord envisagés comme des ruptures ou des coupures par rapport au traitement récurrent d'un sujet. De plus, comme le souligne Sophie Moirand (ibid. : 4), « un fait ou un événement ne constitue un moment discursif que s'il donne lieu à une abondante production médiatique et qu'il en reste également quelques traces à plus ou moins long terme dans les discours produits ultérieurement à propos d'autres événements ». Si la vague de suicides dans les grandes entreprises publiques a constitué un moment discursif, elle a impliqué que les médias multiplient l'information sur le phénomène de burn out. À travers une étude de cas relative au quotidien Le Monde, nous souhaitons interroger le traitement médiatique de ce phénomène et nous demander si la récurrence qui lui est propre conduit à une formulation fonctionnant non seulement dans le cadre de la construction discursive de l'information mais aussi comme un figement relevant d'un ordre discursif (KriegPlanque, 2012 : 98). Quels sont les différents moments discursifs spécifiques à l'usage du terme « burn out » dans Le Monde ? Comment sont-ils caractérisés? En quoi conduiraient-ils à un figement? Quels en sont les enjeux du point de vue du traitement socio-politique du phénomène par la presse écrite?

Pour tenter de répondre à cette problématique, un corpus - dont le détail sera explicité ci-après - a été dégagé à partir de la collecte d'archives de la presse écrite papier et web $^{3}$. Les méthodes appliquées sont celles de l'analyse

' Plus généralement situé comme un syndrome d'épuisement professionnel, le burn out est caractérisé par plusieurs types de symptômes : la perte du sens du travail et de l'adaptabilité, l'irritabilité et divers troubles somatiques comme des tensions musculaires. II est à distinguer de la dépression dans la mesure où il se manifeste moins par une tristesse que par un épuisement. En 20I5, on envisage, en France, qu'il touche 5 à $10 \%$ de la population active (Zawieja, 2015 : 9).

2 L'hebdomadaire Télérama reprend le terme de « mode », utilisé par Didier Lombard, pour en formuler un titre le 23 septembre 2009 : « De la mode au suicide ».

${ }^{3}$ Les données ont été recueillies jusqu'au printemps 2015 qui correspond à la période de la discussion du projet de loi « Rebsamen » (loi relative au dialogue social et à l'emploi, promulguée le I8 août 2015) durant laquelle la question de la reconnaissance du burn out comme maladie professionnelle s'est posée. 
des discours, afin d'étudier la construction discursive de l'information (Ringoot, 2014), et de l'analyse lexicométrique (Déjean, Ratinaud, 2009) concernant Le Monde, ici à partir de l'occurrence « burn out ». Nous relatons les caractéristiques du corpus pour en situer l'éventuel traitement socio-politique.

Sur ce point, nous reprenons les travaux d'Alain Rabatel sur la médiatisation des suicides à France Télécom, notamment ceux publiés dans Questions de communication (20 I I). L'auteur s'est plus particulièrement penché sur les articles rédigés à ce sujet en France, durant l'année 2009. II met en évidence la manière dont cette médiatisation a évolué, depuis le traitement du fait divers à celui du fait de société. En effet, il expose le passage d'une approche psychologisante des causes du suicide dans l'entreprise à une mise en cause progressive de l'organisation du travail (Rabatel, 20 l0, 20l I). Du point de vue formel, cela induit le glissement d'un traitement émotionnel du fait vers la mise en évidence de signes saillants d'une série pouvant être intégrée dans l'histoire du management contemporain. Toutefois, Alain Rabatel conclut sur le fait que cette mise en évidence de la logique entrepreneuriale ne va pas jusqu'à la critique ou la dénonciation des transformations néo-libérales du capitalisme. Ainsi repartons-nous de ces conclusions établies à partir d'un corpus de 2009. Cependant, notre perspective est moins synchronique que diachronique, dans la mesure où les premières archives traitées datent de 1997.

L'intérêt de la démarche d'Alain Rabatel est de mettre en évidence les enjeux de la médiatisation, en particulier dans la construction du débat public sur un phénomène social. Nous souhaitons adopter son point de vue critique. En effet, si les médias s'emparent du sujet à travers le prisme du fait divers ou du fait de société, la définition du burn out n'est pas fixée ${ }^{4}$, notamment du point de vue médical. Le burn out n'est pas mentionné dans les classifications internationales des troubles (CIM- I0, DSM-5). Ses acceptions sont donc variables. S'il s'agit d'un syndrome d'épuisement professionnel, comme le souligne Philippe Zawieja (2015 : 17), le processus auquel il conduit serait dû à l'écart entre les réalités du travail et les représentations que les individus s'en sont faites. L'auteur de Burn out (Zawieja, 20 I5), insiste sur le fait qu'en tant que risque psycho-social, une approche globalisante du phénomène, permettant d'envisager la souffrance au travail d'abord comme une souffrance sociale, est la plus pertinente. Telle est aujourd'hui l'approche proposée par la Théorie critique que nous souhaitons adopter pour ces raisons critiques.

Dans une première partie, notre ancrage théorique se situe alors dans le point de vue de l'École de Francfort sur les « pathologies sociales ». Nous verrons d'abord comment la notion pourrait être intégrée dans le débat public. L'analyse du monde du travail dans les pays capitalistes et des discours qui y sont véhiculés permettrait de situer des causes du burn out. II y a différentes représentations socio-historiques de la fatigue psychique, par exemple la mélancolie au Xvle siècle ou le spleen romantique au XIXe. Le burn out a aujourd'hui ceci de spécifique que le terme utilisé

\footnotetext{
${ }^{4}$ Le terme de burn out est plus fréquemment mentionné sans italiques : burn-out. Nous y ajoutons des guillemets dans la restitution de l'analyse lexicale.
} 
pour désigner ce type d'épuisement cristalliserait une préoccupation sociétale plus large, en particulier depuis la généralisation du salariat à partir de la deuxième moitié du $x x^{e}$ siècle (ibid.). Pour comprendre ensuite la manière dont il est médiatiquement situé, ainsi que sa récurrence et sa reprise, dans une deuxième partie, nous mettrons en évidence la nature des relais d'informations, la fréquence du sujet, afin de situer les titres de presse écrite qui traitent le plus du phénomène. Dans une troisième partie, plus développée, nous évoquerons l'analyse de publications du Monde comme étude de cas.

\section{Les significations socio-politiques du burn out}

Comme l'indique Philippe Zawieja (ibid.), la Théorie critique constitue une des rares approches globalisantes permettant de circonscrire des significations socio-politiques du burn out. Ainsi la philosophie sociale - dans laquelle Axel Honneth (2006) l'inscrit désormais - suppose-t-elle une attention particulière aux « pathologies sociales 》. Elle relève de la philosophie en tant qu'elle repose sur des présupposés normatifs concernant une vie normale, c'est-à-dire sur « les conditions de l'autoréalisation de l'être humain » (ibid. : 87). Elle se distingue de la sociologie et se rapporte à cette tradition dont Axel Honneth est héritier, celle de la Théorie critique par sa prétention à discerner ces pathologies sociales. En amont, elle repose sur une morale de la reconnaissance (Honneth, 1975) propre à l'École de Francfort telle que l'auteur entend la poursuivre après Jürgen Habermas. En aval, cette philosophie est sociale en tant qu'elle porte sur des processus de déformation pathologique du social.

\section{Le burn out comme pathologie sociale}

Pour Axel Honneth, dans La Société du mépris (2006), les pathologies sociales sont situées grâce à l'analyse des structures générales d'une vie « réussie » (la réalisation de soi des êtres humains). Une pathologie est « un dysfonctionnement organique que le diagnostic doit révéler ou déterminer » (ibid : 87). Elle s'oppose à ce qui est normal. Si, dans un contexte social, la normalité est toujours déterminée culturellement, elle peut cependant se définir suivant « les conditions qui garantissent aux membres de cette société une forme inaltérée de réalisation de soi » (ibid: 88). Ainsi, ce qu'Axel Honneth entend par « pathologies sociales » doit s'entendre comme « des relations ou des évolutions sociales qui portent atteinte, pour nous tous, aux conditions de réalisation de soi » (ibid. : 179, voir aussi Heller, 2009). En amont, la préoccupation du philosophe pour les « pathologies sociales » repose sur une réflexion morale (une vie réussie ou une autoréalisation de soi tributaires de la reconnaissance). Leurs prolongements sont politiques dans la mesure où elles conduisent non seulement à s'interroger sur la justice, mais aussi, de manière plus concrète, aux conséquences de l'évolution du capitalisme néo-libéral. 
Si l'optique d'Axel Honneth demeure francfortienne, l'auteur se détache des perspectives offertes par Jürgen Habermas. En effet, il substitue au paradigme de la communication, un paradigme de la reconnaissance. La raison en est la suivante. Les conditions rationnelles d'une entente, comme les envisage Jürgen Habermas, supposent que les individus - pour y parvenir - soient égaux en droits et aient acquis une identité sociale. Or, ceux-ci peuvent rencontrer des problèmes d'identité et ces problèmes sont l'indice d'un « développement pathologique de la société » (Honneth, 2006 : 196). Les conditions communicationnelles de l'entente sont donc déterminées par la formation réussie de l'identité de chacun. Cette formation est dépendante de trois types de reconnaissance : dans les relations intimes (l'amour et l'amitié), dans la société (la sphère du droit) et enfin celle qui concerne l'appréciation des prestations et capacités des individus. L'estime sociale découle de ce troisième type de reconnaissance. Pour Axel Honneth (ibid : 197), elle provient du « travail formellement organisé ». Dans La Société du mépris, l'auteur ne donne pas l'exemple du burn out. II s'intéresse, par exemple, aux conséquences de la nonrémunération du travail ménager. II en conclut que la formation réussie de l'identité et l'expérience de la reconnaissance sont dépendantes de l'organisation et de la répartition du travail. Cependant, en lien avec le paradigme de la reconnaissance, l'analyse du monde du travail dans les pays capitalistes d'Axel Honneth peut nous permettre de comprendre des causes du burn out.

\section{Les causes socio-politiques du burn out}

L'auteur de La Société du mépris distingue son paradigme de la reconnaissance de ce qu'il nomme « l'idéologie de la reconnaissance » (ibid : 269). Dans le monde du travail des pays capitalistes, règne désormais cette idéologie véhiculée par des discours sur l'autoréalisation de soi. II y a une nouvelle manière de s'adresser aux employés; la littérature managériale n'évoque plus « les salariés », mais « les travailleurs-entrepreneurs ». Cette nouvelle dénomination a plusieurs caractéristiques. La première est que tout changement (d'emploi, de contrat de travail, etc.) est conçu comme le fruit d'une décision de l'individu. La seconde caractéristique est que, comme un entrepreneur, tout travailleur est flexible, créatif et autonome. Ainsi l'idéologie de la reconnaissance génère-t-elle un nouveau rapport à soi qui implique d'assumer volontairement des charges de travail toujours plus importantes. La dénomination « travailleur-entrepreneur » crée les conditions visant à se transformer soi-même en marchandise. Axel Honneth écrit plus précisément que « la flexibilisation et la dérégulation du travail qui accompagnent les transformations néo-libérales du capitalisme exigent des capacités à se transformer soi-même en marchandise à des fins productives, que la dénomination valorisante d'« entrepreneur de soi-même » contribue précisément à créer » (ibid : 270). Enfin, les dispositions institutionnelles qui permettraient une réalisation effective des valeurs telles que celles de flexibilité, de créativité et d'autonomie ne sont pas garanties. La promotion de ces valeurs n'est donc 
pas assortie de changements permettant de la rendre effective. Eu égard à ces conditions de travail inchangées, les individus ne peuvent que simuler la motivation, la flexibilité et les compétences.

Cette désinstitutionnalisation a une autre conséquence, celle de conduire à une surcharge qui a elle-même des effets relatifs à l'élévation du rythme de vie des individus touchant tous les domaines: la technique, les transports, la communication et la production. Ainsi un autre facteur essentiel de cette « pathologie sociale » qu'est le burn out relèverait, selon une analyse francfortienne, de l'accélération du rythme de vie. Dans Accélération. Une critique sociale du temps, Harmut Rosa (2005) évoque le fait que, malgré l'augmentation du temps libre, un stress est inexorablement consécutif à cette accélération. En effet, les individus éprouvent un sentiment de pression temporelle qui aurait deux facteurs : la peur de manquer quelque chose et la contrainte d'adaptation. Concernant le premier facteur, la peur de manquer quelque chose d'intéressant est en quelque sorte le résultat d'un projet culturel, à l'échelle de la société, valorisant la multiplication d'expériences. Les individus eux-mêmes veulent vivre plus vite. Suivant le deuxième facteur, la contrainte d'adaptation, ils sont obligés de vivre plus vite à cause de l'accélération de la transformation sociale (ibid.: I 67). Même s'ils décident de prendre du temps, le monde autour d'eux continue à évoluer. L'impossibilité de soutenir des valeurs telles que la flexibilité, la créativité et l'autonomie grâce à des dispositions institutionnelles, ainsi que l'accélération du temps, génèrent de multiples souffrances au travail (Clot, 2008 ; Dejours, 2008). Parmi ces souffrances, le burn out, syndrome d'épuisement professionnel, en est aujourd'hui une des plus significatives. II peut être considéré comme une pathologie sociale.

Le thème de la souffrance au travail a été introduit dans le débat public, en France, par les médias suivant une approche psycho-sociale. Comme le souligne David Douyère (20|4), ceux-ci l'ont plus particulièrement fait consécutivement aux suicides dans les grandes entreprises nationales, en convoquant des experts et des témoins 5 . Cette médiatisation a certes eu pour effets d'informer les salariés de ces mêmes entreprises sur ce qu'il se produisait en interne et d'engager les membres dirigeants de ces organisations dans une communication de crise (ibid.) sur la souffrance au travail. Cette dernière a renvoyé à un objet public investi d'un point de vue socio-discursif. En effet, les salariés ont repris les discours véhiculés par les médias pour désigner les réalités qu'ils vivaient eux-mêmes ou les affects ressentis. D'un autre côté, les dirigeants ont fini par prendre la parole pour produire un discours alternatif, en réponse à celui présenté par les journalistes (ibid.). L'évocation de la souffrance au travail suivant une approche psycho-sociale a pu avoir des conséquences sur l'usage du lexique qui lui est lié. C'est plus spécifiquement celui du terme de burn out que nous souhaitons d'abord restituer au regard de sa récurrence et de sa reprise dans les médias de presse écrite.

\footnotetext{
5 Dans le cas des suicides à France Télécom, Alain Rabatel $(2010: 34)$ met en évidence que les experts ont été sollicités tardivement ; ils sont peu consultés dans la période de mai à mi-août 2009.
} 


\section{Le contexte médiatique du traitement du phénomène de burn out par la presse écrite}

\section{Description du corpus : l'hétérogénéité temporelle}

Le corpus a été construit à partir de la base de données Europresse pour la presse écrite papier et les sites web de journaux en ligne 6 . Les données ont été recueillies jusqu'au 8 avril 2015. Pour la presse écrite papier, n'ont été retenus que les titres pour lesquels on dénombrait plus d'un article. En ce qui concerne plus spécifiquement les titres nationaux, quotidiens et hebdomadaires, les dates de premières archives varient entre les années 2004 et 201 I (tableau I).

Tableau I. Archives de la presse nationale

\begin{tabular}{|c|c|}
\hline Titres (par ordre alphabétique) & Dates de la première archive sur Europresse \\
\hline Aujourd'hui en France & $22 / 1$ I/2010 \\
\hline La Croix & $01 / 12 / 2011$ \\
\hline Le Figaro & $19 / 12 / 2005$ \\
\hline Le Point & $24 / 06 / 2004$ \\
\hline L'Express & $\mid 8 / 01 / 2012$ \\
\hline L'Humanité & $22 / 11 / 2010$ \\
\hline Libération & $26 / 03 / 2008$ \\
\hline Marianne & $02 / 04 / 2011$ \\
\hline Valeurs actuelles & $\mid 4 / 04 / 2011$ \\
\hline
\end{tabular}

Pour la presse régionale, les dates de premières archives s'étalent encore davantage dans le temps puisqu'elles varient de 1997 pour Le Progrès et 20 I4, pour L'indépendant et Charente libre (tableau 2).

Tableau 2. Archives de la presse régionale

\begin{tabular}{|c|c|}
\hline Titres (par ordre alphabétique) & $\begin{array}{c}\text { Dates de la première archive } \\
\text { dans Europresse }\end{array}$ \\
\hline Charente libre & $14 / 06 / 2014$ \\
\hline La Nouvelle République (Centre-Ouest) & $23 / 06 / 2007$ \\
\hline La Voix du Nord & $09 / 09 / 2004$ \\
\hline Le Bien Public (Bourgogne) & $29 / 09 / 2008$ \\
\hline
\end{tabular}

\footnotetext{
${ }^{6}$ Le traitement des données relatives au corpus de presse écrite papier et web, portant sur le burn out, est mentionné dans un chapitre co-écrit avec David Douyère (Chevret, Douyère, 2016 : | 89-191).
} 


\begin{tabular}{|c|c|}
\hline Le Journal de Saône-et-Loire & $29 / 08 / 2006$ \\
\hline Le Parisien & $01 / 10 / 2013$ \\
\hline Le Progrès (Lyon) & $05 / 01 / 1997$ \\
\hline Le Télégramme (Bretagne) & $02 / 12 / 2002$ \\
\hline L'Est Républicain & $13 / 12 / 2011$ \\
\hline L'indépendant (Pyrénées) & $06 / 02 / 2014$ \\
\hline Midi Libre & $12 / 10 / 2011$ \\
\hline Ouest-France & $21 / 05 / 2012$ \\
\hline Paris-Normandie & $19 / 04 / 2008$ \\
\hline Presse Océan (Vendée) & $05 / 10 / 2011$ \\
\hline Sud-Ouest & $10 / 10 / 2005$ \\
\hline
\end{tabular}

Concernant les données sur l'internet, nous avons retenu les quatre Pure players parmi les plus connus (Atlantico, Le Journal du net, Médiapart et Rue89) à la date du 9 mars 2015.

\section{Analyse du corpus : I'hétérogénéité dans le traitement du sujet}

Figure I. Nombre d'articles par titre de la presse nationale

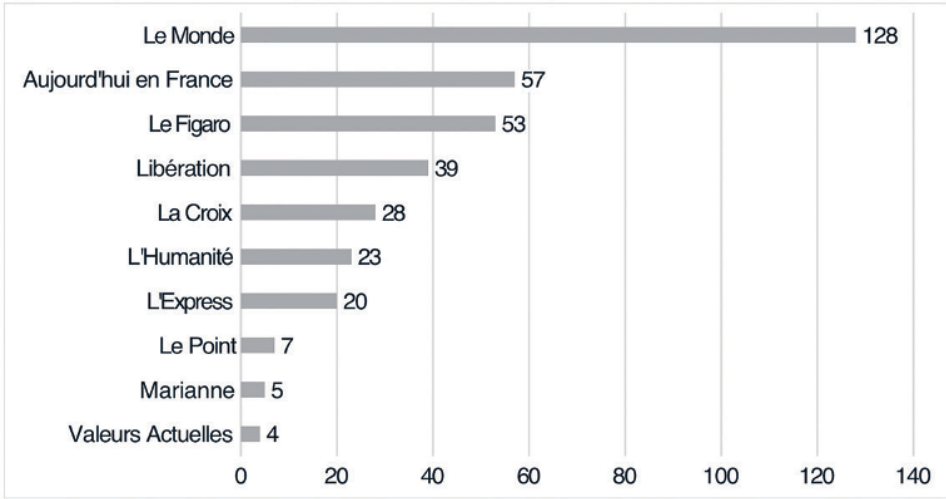

On observe l'importance de traitement d'abord pour Le Monde puis pour Aujourd'hui en France qui est l'édition nationale du Parisien, ensuite pour Le Figaro et Libération (figure I). Le burn out est un sujet davantage traité par la presse quotidienne que par la presse magazine. On peut y voir l'objet d'une interrogation. En effet, par distinction avec le suicide qui peut en être une conséquence, le phénomène n'est pas réductible au fait divers et à la construction d'un événement suite à la réception d'une dépêche reçue par un journaliste de presse quotidienne (Rabatel, 20l0). II peut être envisagé par la presse magazine comme motif à la constitution d'un dossier permettant d'identifier ce phénomène émergent (Charon, 1999 : 80). 
Figure 2. Nombre d'articles par titre de la presse régionale

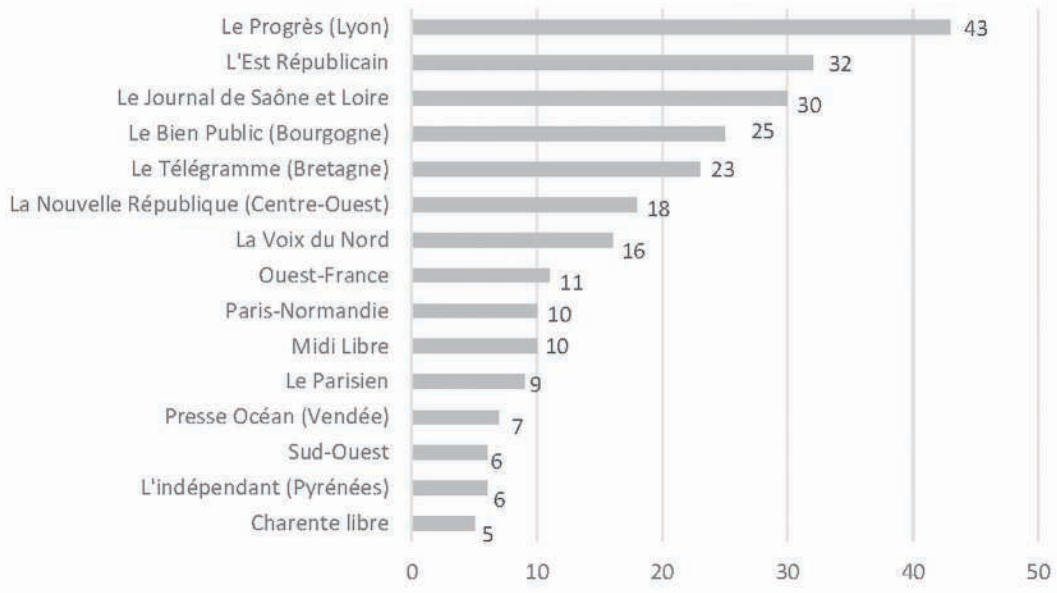

Dans la presse quotidienne régionale, le traitement du sujet est l'objet d'une grande hétérogénéité numérique. Cette variation de traitement pourrait être corrélée avec le public destinataire des informations. Par exemple, on voit que le nombre le plus important d'articles se situe dans Le Progrès (figure 2). Ce dernier est un quotidien de Lyon, soit la troisième ville de France là où le phénomène peut probablement se produire. À partir de cette déduction, on peut s'étonner de l'importance du traitement par le Journal de Saône et Loire qui couvre une région où la population vit davantage en zone rurale. À l'instar d'Alain Rabatel (2010, 20I I), il est aussi possible de considérer que le sujet s'étant imposé suivant une problématique sociétale, il est susceptible de toucher un large public populaire, lecteur de la presse quotidienne régionale.

Figure 3. Nombre d'articles par Pure player

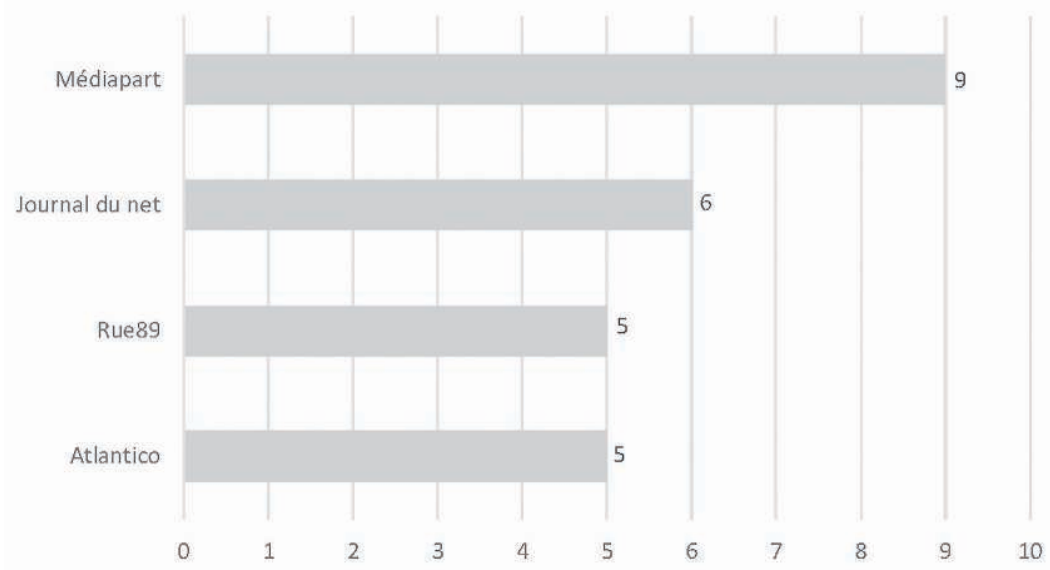


Le sujet du burn out n'est pas beaucoup traité par les Pures players. Même le chiffre relatif à Médiapart peut sembler peu significatif dans la mesure où il contient pour moitié les occurrences relatives aux blogs hébergés par la plate-forme. Les archives ne couvrent pas des périodes comparables dans la mesure où certains Pure players sont plus récents que d'autres, c'est notamment le cas d'Atlantico (20 I I).

\section{Caractéristiques dégagées de l'étude du corpus de presse écrite}

Mis à part trois Pure players (Atlantico, Le Journal du Net et Médiapart), on observe l'importance du témoignage et du portrait quand il s'agit d'évoquer le burn out? Mentionnons notamment la récurrence de l'usage des déictiques en particulier du pronom personnel « je »,y compris dans des titres d'information politique et générale : par exemple, « Je suis allée droit dans le mur », La Croix (I5/0I/20 I3), « J'ai poussé trop loin la machine », L'Express (22/0 I/20 I4), « Je vais péter les plombs ! », L'Express (0I/04/20 I5). Cet usage du déictique, ici du pronom personnel « je », permet de voir que le burn out est un mal dont on a conscience, qu'il est possible de nommer. Cette personnalisation a une vertu, celle de rendre le phénomène moins abstrait. Elle a aussi pour finalité de rendre sensible au lecteur la souffrance de celui qui en est victime. Le récit personnel construit une relation directe avec le public. La modalité énonciative suppose d'inclure le lecteur dans ce récit. Ce dernier fait cependant ici obstacle à la possibilité de saisir des enjeux socio-politiques. Le traitement médiatique conduit à esquiver la logique sociale au profit d'une approche psychologisante.

La seconde caractéristique est liée à une rupture dans le traitement du burn out. Cette rupture est trop récente pour en déterminer une conclusion définitive. Cependant, suite à l'appel de députés, paru dans Le Journal du dimanche du 6 décembre 20 I4, il a été question de la reconnaissance du burn out comme maladie professionnelle et de la responsabilité imputable à l'employeur. Le débat dans l'espace public qui s'en est suivi a eu pour conséquence, d'un point de vue discursif, une cristallisation. La médiatisation du débat public sur la reconnaissance du burn out comme maladie professionnelle pourrait conduire à une cristallisation d'enjeux socio-politiques et surtout que l'expression revête un caractère de formule, c'est-à-dire qu'elle soit comme « un ensemble de formulations qui, du fait de leurs emplois à un moment donné et dans un espace public donné, cristallisent des enjeux politiques et sociaux que ces expressions contribuent dans le même temps à construire » (Krieg-Planque, 2012 : I I0).

L'analyse du corpus de presse écrite permet de dégager l'intérêt de faire un point focal sur un titre se dégageant des autres productions écrites. Le choix du Monde

7 Du point de vue de la méthode, le traitement des données issues des médias de presse écrite a tout d'abord permis de situer la quantification de l'occurrence « burn out ». Il a donné la possibilité de dégager des tendances dominantes. L'analyse interroge ensuite l'occurrence du point de vue du genre et, par exemple, de la titraille (Ringoot, 20I4). 
pour une analyse de contenu plus approfondie s'est imposé pour deux raisons. D'abord, comme l'a mis en évidence l'analyse quantitative, c'est dans ce quotidien que le sujet a été le plus évoqué. Ensuite, la manière d'y aborder l'information politique et générale, depuis 1944, laisse supposer que le journal traite d'enjeux socio-politiques du phénomène. Pour situer ces enjeux, se pose la question de la référenciation du burn out. Pour y répondre, il s'agit de procéder à des analyses lexicométriques afin de l'appréhender dans un contexte lexical, puis d'effectuer des analyses de discours permettant de circonscrire ce qui est mesuré, dans un contexte discursif.

\section{Les « moments discursifs » relatifs au « burn out » dans Le Monde}

\section{Description des éléments de corpus : l'année 2009 comme rupture}

Les éléments de corpus relatifs au quotidien Le Monde ont été délimités à partir de la consultation des archives du journal depuis sa fondation en 1944 entrecroisée avec celle de la base de données Europresse. Ils ont été circonscrits à partir des premiers usages du terme au sens de syndrome d'épuisement professionnel et de leur récurrence, c'est-à-dire après le 3 I janvier 2003 ( 128 articles). On notera cependant que la première évocation du syndrome date du 12 janvier 1985 et concerne les risques encourus par un célèbre joueur de tennis de l'époque, John McEnroe. On peut également souligner qu'avant 2003 l'expression désigne une pratique qui consiste à faire chauffer les pneus d'une motocyclette ou d'une voiture jusqu'à leur éclatement ou à brûler les freins. Enfin, une autre caractéristique est qu'on ne décompte que 13 articles entre 2003 et 2009 contre II 5 entre 2009 et 20I5. L'année 2009 - qui correspond au sommet de la vague de suicides à France Télécom - est donc bien une année charnière.

\section{Analyse des éléments de corpus : des visées informatives et explicatives}

L'analyse des éléments de corpus a alors été réalisée à partir des différents genres journalistiques. Comme Le Monde est une publication classée dans la presse d'information politique et générale, nous reprenons l'information comme catégorie textuelle de ces genres tels qu'ils sont circonscrits par Ernst-Ulrich Grosse (200I) : la brève, le filet, la tribune, l'enquête, l'analyse, le reportage, l'interview, le portrait et la critique. 
Figure 4. Genres dans Le Monde (3I janvier 2003-9 mars 20I5)

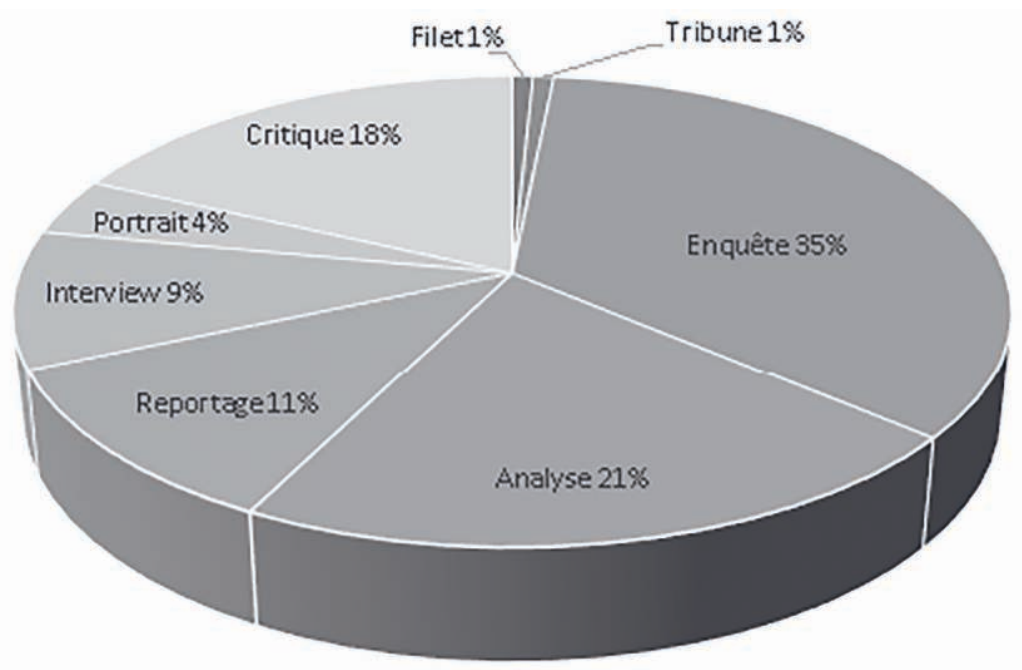

- Filet - Tribune - Enquête -Anayse
- Reportage - Interview - Portrait - Critique

Relevons que l'importance du nombre de critiques (I $8 \%$ ) (figure 4) s'explique par le fait que l'édition du Monde des Livres paraissant le vendredi est bien distincte. II s'agit, pour l'essentiel, de critiques de livres portant sur le sujet du burn out. On voit que les formats courts du type « filet » sont presque inexistants, voire absents comme la brève. En revanche, avec respectivement 35 et 21 \%, l'enquête et l'analyse sont les genres les plus fréquents. Les visées seraient donc plutôt explicatives du phénomène de burn out. Pour comprendre le traitement médiatique de ce phénomène par Le Monde, une analyse statistique du lexique est effectuée pour situer l'occurrence «burn out » dans son contexte lexical ; une analyse de discours donne ensuite la possibilité de la circonscrire dans son contexte discursif.

D'abord, l'analyse lexicométrique des accroches ${ }^{8}$ du journal tend effectivement à montrer que les stratégies énonciatrices sont informatives et explicatives. Elle a été faite à l'aide du logiciel Interface de R pour l'Analyse multidimensionnelle des textes et des questionnaires (Déjean, Ratinaud, 2009). Grâce à l'analyseur ALACESTE $^{9}$ (Reinert, 1983, 2008), le texte à étudier est découpé ; la fragmentation qui s'ensuit donne des unités de contexte élémentaires (UCE). Le logiciel libre et gratuit

\footnotetext{
${ }^{8}$ En répondant à « Qui ?, Quoi ?, Où ?, Quand ?, Comment ?, Pourquoi ? », les accroches dévoilent les stratégies énonciatrices du journaliste dans la mesure où elles constituent à la fois des résumés des articles et des incitations à la lecture (Mouriquand, 20 I I : I |2).

9 ALCESTE :Analyse des lexèmes cooccurrents dans un ensemble de segments de textes. Les cooccurrences sont des catégories de description statistiques ; leur traitement informatisé permet de les situer.
} 
développé par Pierre Ratinaud (I RaMuTeQ, http://www.iramuteq.org, Université Toulouse 3, Lerass) permet d'effectuer une analyse factorielle des correspondances (AFC) (Benzécri, 1982) entre des formes. Dans la figure ci-dessous (figure 5), les formes les plus imposantes sont statistiquement les plus fréquentes. Parmi elles, on compte ainsi professionnel, travail et français.

Figure 5. Répartition lexicale relative au corpus du Monde (analyse factorielle des correspondances)

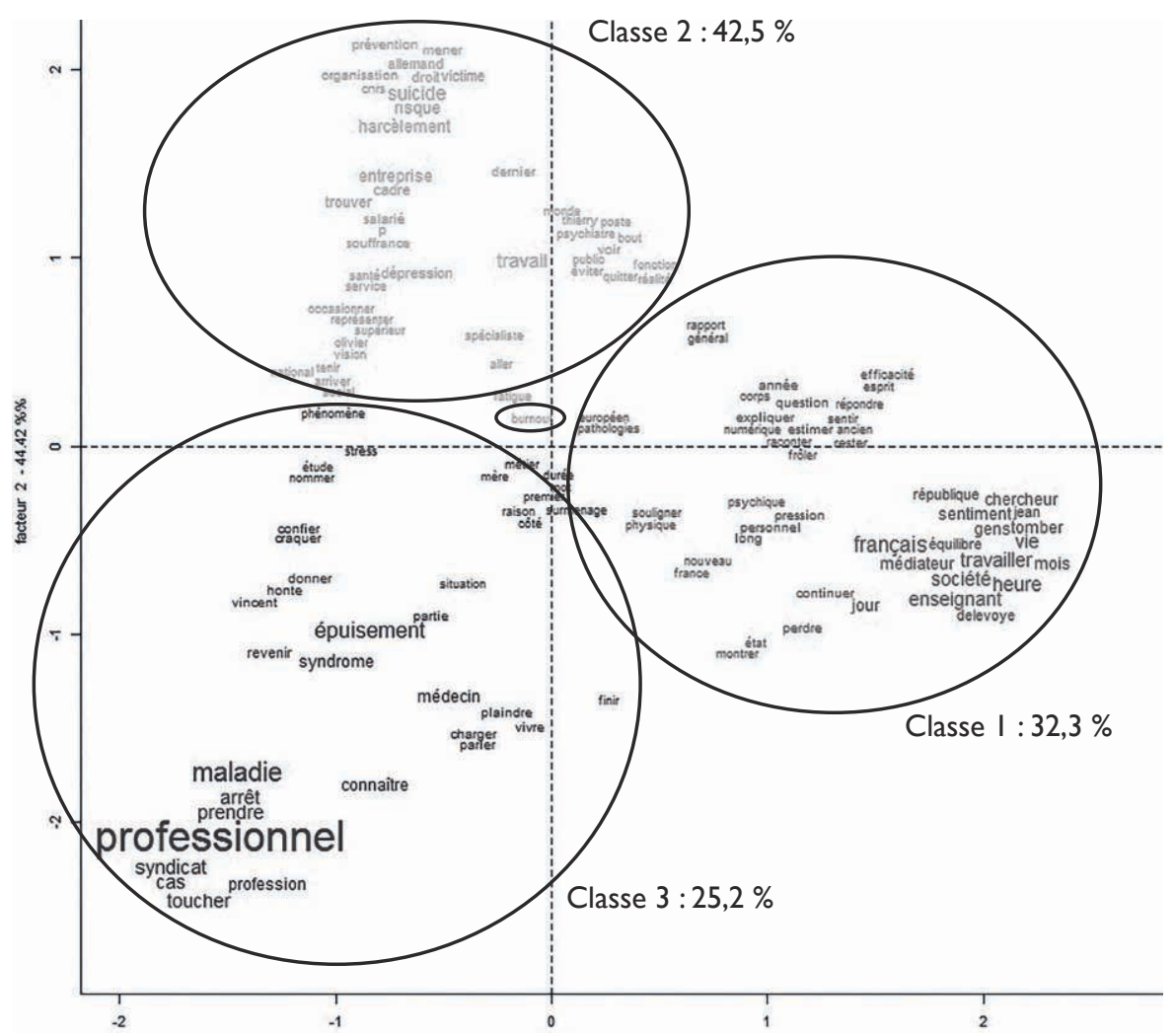

Ainsi se dessinent trois classes. Ces dernières sont de taille inégale. Avec 42,5 \% des formes, la classe 2 (verte), celle relative au travail, est la plus importante d'un point de vue statistique ; viennent ensuite les classes I (rouge), avec 32,3\% concernant le contexte, et 3 (bleue), avec 25,2\%, qui porte sur le risque psycho-social. Le terme « burn out », les causes et les conséquences, se trouve dans la classe 2 (verte). La classe 3 (bleue) peut être rapportée au risque psycho-social en lui-même ; elle contient également les réalités ressenties et vécues par les individus (par exemple, « craquer », « parler », « honte », « plaindre »), mais aussi des réalités situées en dehors du monde professionnel, comme celles subies par une « mère ». Dans la classe I (rouge), sont classés les éléments propres au contexte y compris temporel (« heure », « mois », « jour »). 
Plusieurs caractéristiques discursives peuvent ensuite être dégagées grâce à une analyse qualitative. Les enquêtes sont conduites auprès de professionnels ; il s'agit de témoigner plus spécifiquement des souffrances des médecins hospitaliers, des policiers, des enseignants et des chercheurs. Hormis les fonctionnaires très largement interviewés, on dénombre un article sur les banquiers et un autre sur les artistes. Le secteur médical qui fait l'objet du nombre le plus important d'enquêtes est très largement au-dessus d'autres catégories socio-professionnelles ( 13 articles). Le choix des enquêtes véhicule ici des représentations sociales. Comme le souligne Philippe Zawieja (2015 : 17), le burn out a longtemps été considéré comme une pathologie affectant des professionnels dont le métier est perçu comme une vocation à l'instar du médecin, du policier, de l'enseignant et du chercheur.

Entre 20 I I et 20 I 5, nous observons, dans ces publications du Monde, que des entités collectives sont également nommées. Parmi ces entités, des entreprises sont citées, comme France Télécom (Le Monde, 12/05/20I I), mais aussi des institutions, par exemple l'Université (Le Monde, 07/05/20 I4). Le phénomène concerne également des entités abstraites ; par exemple, « Des titres comme le Nouvel Observateur ou Marianne sont au bord du burn out » (Le Monde, 27/02/2014). II est par ailleurs envisagé comme touchant l'ensemble de la société : «Ce week-end, dans le Journal du dimanche, le psychiatre Boris Cyrulnik a expliqué que la France n'était pas dépressive ; tordant le cou à la société en burn out décrite par le médiateur de la République » (Le Monde, I3/04/20 I I). Ainsi, l'expression « burn out » fait l'objet d'un glissement sémantique, renvoyant ainsi à toute situation de crise, de faillite ou de chaos. Si l'usage de l'anglicisme est en lui-même problématique eu égard au flou sémantique qu'il induit, ce glissement contribue à s'interroger sur le sens de l'énonciation. Pour analyser ce glissement, nous reprenons le détail des différentes périodes marquées par des ruptures, des coupures dans le traitement médiatique du phénomène par Le Monde.

\section{Analyse lexicométrique des « moments discursifs »}

Le texte constitué à partir des articles du Monde est ensuite formaté autour de quatre variables : avant 2003, 2003-2009, 2009-20 I I, 20 I I-20 I 5. Nous ne reviendrons pas sur la période antérieure à 2003 dont l'analyse factorielle des correspondances met en évidence le peu d'intérêt eu égard à l'hétérogénéité discursive des éléments de corpus.

La forme « burnout $»^{10}$ se situant, suite à l'analyse factorielle des correspondances (AFC), à la jonction des abscisses et des ordonnées de la factorisation, la visualisation permet de situer immédiatement les autres formes correspondantes. Sur la période 2003-2009, celles relatives à « burnout » sont : « syndrome », « professionnel », 《 épuisement 》, 《 médecin », 《 dépression 》, 《 stress 》, 《 travail 》, 《 maladie » et « entreprise ». Elles renvoient à la tentative de définition du phénomène (un syndrome d'épuisement professionnel), son contexte d'apparition (l'entreprise)

\footnotetext{
10 Nous avons retenu l'orthographe « burnout » pour l'analyse lexicométrique, la scission en deux de l'expression parasitant les résultats.
} 
sa qualification (une maladie), à ses causes (le stress lié au travail), ses symptômes éventuels (la dépression). La référence au médecin n'a pas nécessairement de liens avec l'idée de solutions thérapeutiques au syndrome d'épuisement professionnel. En effet, le soignant peut également être victime du burn out.

Sur la période 2009-20 I I, des formes réapparaissent: « professionnel », « épuisement », « médecin », « maladie », « dépression », « travail ». De nouveaux termes émergent: 《 année », « français », « suicide », mais aussi « mère ». Cette terminologie renvoie aux suicides advenus à France Télécom durant l'année 2009 et au syndrome d'épuisement vécu par les mères de famille. On note aussi une série de verbes qui n'existait pas dans les correspondances précédentes : « aller 》, « voir », « pouvoir », « faire ». La présence de ces verbes relève de la lemmatisation, c'est-à-dire ici de l'opération qui consiste à ramener les verbes à l'infinitif, effectuée par le logiciel, mais il est à noter qu'elle se caractérise par un usage de performatifs. Ainsi cela implique-t-il d'être passé d'une définition du phénomène en vue d'informer à des préconisations dont la finalité relève de la prévention, par exemple au travers des conseils donnés par un psychiatre (Le Monde, 09/09/2010).

Sur la période de 201 I à mai 2015, apparaissent « entreprise », « travail », « faire », « médecin », « stress », mais aussi « français ». Ces formes existaient préalablement dans les articles, elles deviennent plus fréquentes. II est cependant à noter qu'« entreprise » et « travail » représentent celles les plus proches de « burnout ». Comme le note Alain Rabatel, la médiatisation du phénomène dans la presse écrite a eu cette inflexion, à la fin de 2009, d'imputer plus directement des causes à l'entreprise.

\section{Des différents « moments discursifs » au figement}

Situons cette analyse statistique par rapport aux contextes des différents contenus. La première est qu'il y a distinctement plusieurs « moments discursifs », c'est-à-dire des ruptures qui peuvent ici entrer en relation avec un fait donnant lieu à une production médiatique (Moirand, 2007) opérant des coupures par rapport au traitement récurrent sur la période (professions touchées, causes et solutions). Le premier moment qui permet de comprendre la forme « mère » présente dans l'analyse lexicométrique, porte sur le «burn out maternel», en 201 I : « Les enfants crient parce qu'lls ne savent pas attendre et n'ont pas mis en place une autonomie psychique. Les mères en font trop, et ils demandent toujours plus. Cet état de fatigue et de frustration peut aboutir à un épuisement physique et psychique, qualifié de "burn out" maternel » (Le Monde, 2 I/03/20 I I). Le sujet est ensuite repris dans d'autres titres de la presse : « Ces mères qui n'osent pas avouer leur souffrance » (Marianne, 02/04/20 I I) et « Les mères parfaites se rebiffent 》 (L'Express, 08/06/20 I I). La répétition et la reprise sont ici constitutives d'un « moment discursif »" et elles renvoient à une circulation.

\footnotetext{
" L'expression de « burn out maternel » a d'abord été utilisée notamment par La Croix (Dossier. La fatigue d'être mère, un mal à combattre. « S'attaquer au mythe de la mère parfaite », I3/04/2005). Cependant, sa reprise dans d'autres titres ne s'observe pas à ce moment-là.
} 
Le deuxième moment a lieu en mars-avril 20I I ; il explique l'importance d'une classe consacrée à « français » qui contient des formes à l'instar de « Delevoye ${ }^{12} 》$, « médiateur » et « société », et concerne le « malaise » vécu dans la société française la dernière année du quinquennat du président de la République Nicolas Sarkozy : "Vous parlez cette fois de "burn out" de la société. II trahirait, selon vous, "un besoin urgent de bâtir de nouvelles espérances". La vie des Français se serait donc encore détériorée? 》) (Le Monde, 22/03/20 I I). Sa reprise, dans un deuxième temps est présentée comme un discours rapporté : «Le burn-out de la société française trahit un besoin urgent de bâtir de nouvelles espérances à la hauteur des efforts fournis. La fébrilité du législateur trahit l'illusion de remplacer par la loi le recul des responsabilités individuelles et de la morale. Le maintien sous perfusion de citoyens assistés permet de soulager nos consciences, mais pas de résoudre nos problèmes 》 (Le Monde, 02/04/20 I I). L'article est un entretien avec Jean-Paul Delevoye, qui quitte alors ses fonctions. La reprise dans la presse écrite (23 et 26 mars 201 I pour Libération et le 29 mars 201 I pour Le Figaro) est consécutive aux résultats des élections cantonales de cette année-là qui voient la montée des partis populistes comme le Front national.

Cependant, selon une première conclusion, l'usage du terme de «burn out » hors contexte professionnel pour désigner le syndrome dont tout type d'entité peut être victime, et sa récurrence, semblent être la conséquence d'un processus inhérent aux « moments discursifs » jusqu'à figer ce terme, à en faire une expression « prête à l'emploi » (Krieg-Planque, 2012 :98). Le terme est désormais figé (Fiala, Habert, 1989). II se caractérise tout d'abord par une structure composée de deux mots courts ${ }^{13}$ facilitant l'usage et renvoyant à une situation historique spécifique où ne règnent plus ni le « spleen » ni la « mélancolie ». La seconde conclusion est ensuite propre aux causes. La perspective diachronique liée à ce travail sur les archives du Monde ne conduit pas à des résultats différents de ceux apportés par Alain Rabatel (20 I0,20 I I) qui a analysé la médiatisation des suicides à France Télécom durant l'année 2009. L'argument de la logique entrepreneuriale comme cause du burn out ne va pas jusqu'à la critique des transformations néolibérales du capitalisme. De plus, il ressort de l'analyse que le burn out est envisagé comme affectant avant tout des individualités. Par exemple, entre 20 I I et 20 I5, on trouve I,5 article par an qui envisage le numérique comme cause du burn out et surtout la responsabilité des individus dans leur incapacité à se déconnecter. Le phénomène serait dû davantage aux individualités qu'au système.

\footnotetext{
${ }^{12}$ Jean-Paul Delevoye est médiateur de la République du I3/04/2004 au 22/06/20 I I.

${ }^{13}$ Le « burn out » est un synthème, au sens de la linguistique fonctionnelle : « Ce qu'il y a de commun à tous les composés et tous les dérivés, c'est d'abord l'unité sémantique du complexe qui est marquée par le fait que chacun correspond normalement à un choix unique. [...] une chaise-longue d'une taille inaccoutumée n'est pas une chaise-plus-longue, mais une chaise-longue plus longue que les autres. [...] Les composés, qu'ils dérivent de figements ou non, et les dérivés sont désignés comme des synthèmes » (Martinet, 1970 : 133).
} 


\section{Conclusion}

Ainsi, Alain Rabatel s'est-il intéressé à un moment discursif ${ }^{14}$, dans le journal Le Monde en 2009 après la vague de suicides à France Télécom, caractérisé par l'émergence des critiques des logiques entrepreneuriales (Rabatel, 2010, 20l I). Notre attention s'est plus particulièrement portée sur les moments discursifs autour de l'expression burn out. Avant le débat public sur la reconnaissance comme maladie professionnelle ${ }^{15}$, le burn out est traité suivant une approche plus sociétale que politique, notamment comme l'étude de cas du Monde le montre, dans le traitement des causes avec l'imputation du phénomène à l'incapacité des individus à se déconnecter du numérique. Même quand les méthodes managériales sont évoquées par les médias de presse écrite, la logique socio-économique en œuvre est peu circonscrite. Si les mécanismes entrepreneuriaux peuvent être dénoncés, le burn out est rarement renvoyé à une « pathologie sociale » (Honneth, 2006) parce que les conséquences du néo-capitalisme ne sont presque pas envisagées. Avant décembre 20 I4, le traitement médiatique par la presse écrite a donc pu, dans une certaine mesure, participer de la même logique que celle qui conduit au burn out, celle consistant à faire peser sur les individus le poids de leurs responsabilités.

Cependant, en 2011, on observe un glissement sémantique, particulièrement intéressant, qui concerne le « burn out maternel ». Ce glissement induit de désigner un syndrome d'épuisement des mères de jeunes enfants et donc des problèmes liés à la pratique de la parentalité. Offrant une certaine plasticité référentielle, l'expression burn out sert à désigner des réalités différentes. Sa récurrence et sa reprise dans les médias en font désormais un signe « polychrésique » (Jeanneret, 20 I $4: 21$ ), c'est-àdire que l'usage de ce signe sert plusieurs enjeux sociaux à la fois, notamment ceux relatifs à la vie au travail, mais aussi sociétaux ${ }^{16}$, ici ceux spécifiques à la famille via le sujet de la pratique de la parentalité. À la lumière de l'analyse du corpus, il semble que l'expression « burn out » revêt un caractère figé ainsi construite : « burn out maternel ». Son caractère figé est une condition pour qualifier cette expression de formule. Son

\footnotetext{
${ }^{14}$ La première rupture dans la médiatisation du burn out, en 2003, avait concerné l'explosion du thème général de la souffrance au travail ; il y a eu 12 ouvrages publiés cette année-là en France, un maximum depuis le traitement médiatique du phénomène (Bourion, 2010 : 109).

${ }^{15}$ La reconnaissance juridique du burn out est à nouveau d'actualité en 2016 , notamment à travers une réappropriation par les hommes politiques de cet objet public. En effet, après le retrait du burn out par le Sénat, le 24 juin 2015, de la liste des maladies professionnelles suite à l'inscription par l'Assemblée nationale des maladies psychiques dans le projet de loi «Rebsamen » sur le dialogue social, le député du Parti socialiste des Yvelines, Benoît Hamon a déposé, le 17 février 2016, une proposition de loi visant à faire supprimer la limite des $25 \%$ de taux d'invalidité qui fait à ce jour obstacle à ce que le syndrome d'épuisement professionnel puisse être reconnu (« Après un burn out, l'étape du retour au travail est cruciale », LeMonde.fr, 22/02/2016). I| est également à l'initiative d'une pétition en ligne (http://petition-burnout.fr/) « Pour la reconnaissance du burnout comme maladie professionnelle » qui réunit 10585 signatures au 27 juin 2016, dont celles de 84 députés.

${ }^{16}$ À travers l'idée d'enjeux sociétaux de l'usage de l'expression « burn out », nous entendons par « sociétal » ce qui est vécu au sein de la société, comme dans l'expression d'« orchestre sociétal » utilisée parYves Winkin (2016).
} 
usage implique d'ailleurs d'autres caractéristiques propres à la «formule » (KriegPlanque, 2009). Comme nous l'avons exposé en évoquant la reprise dans Marianne et L'Express, l'expression «burn out » circule. Elle devient également un référent social eu égard, par exemple, à son appropriation dans les forums de sites web ${ }^{17}$. Enfin, son caractère polémique n'est pas dubitable dans la mesure où le glissement sémantique qu'elle induit peut faire obstacle à sa reconnaissance comme maladie dans le champ professionnel, en particulier à la qualification de « maladie professionnelle » par le droit. Ainsi revêt-elle bien le statut de ce qu'Alice Krieg-Planque appelle une « formule » que son flou sémantique tend à nourrir. Nous souhaitons donc, à la suite de cette étude de cas, défendre l'idée qu'un glissement de la définition de la formule peut revêtir un intérêt, en regard de ce qui a été observé, en particulier en insistant non seulement sur les « enjeux politiques et sociaux 》 (Krieg-Planque, 2009, 20I2) mais aussi sur des enjeux sociétaux.

\section{Références}

Benzécri J.-P., 1982, Histoire et préhistoire de l'analyse des données, Paris, Dunod.

Bourion C., 20 I0, « La souffrance est-elle un signal du ressort du développement personnel ou du ressort du développement providentiel ? », Revue internationale de psychosociologie, 16, 39, pp. 205-226.

Charon J.-M., 1999, La Presse magazine, Paris, Éd. La Découverte, 2008.

Chevret C., Douyère D., 2016, « Les traumas du travail au vu des médias 》, pp. 175-196, in : Zawieja P., dir., Psychotraumatologie du travail, Paris, A. Colin.

Clot Y., 1995, Le Travail sans l'homme, Paris, Éd. La Découverte, 2008.

Déjean S., Ratinaud P., 2009, « IRaMuTeQ : implémentation de la méthode Alceste d'analyse de texte dans un logiciel libre », Rencontres Méthodes et Apprentissages en sciences humaines et sociales/ Accès : http://repere.no-ip.org/Members/pratinaud/mes-documents/articles-etpresentations/presentation_mashs2009.pdf/view.

Dejours C., 1980, Travail, usure mentale. De la psychopathologie à la psychodynamique du travail, Paris, Bayard, 1993, 2000, 2008.

Douyère D., 20 I4, « Médias et Risques Psycho-Sociaux 》, pp. 464-468, in : Zawieja P., Guarnieri F., dirs, Dictionnaire des risques psycho-sociaux, Paris, Éd. Le Seuil/Mines Paris Tech.

Fiala P., Habert B., 1989, « La langue de bois en éclat : les défigements dans les titres de presse quotidienne française », Mots. Les langages du politique, 21, pp. 83-99.

Grosse E.-U., 200I, «Évolution et typologie des genres journalistiques », Semen, 3. Accès : http://semen.revues.org/26I5.

\footnotetext{
${ }_{17}^{17}$ Par exemple, une observation des résultats suite à une exploration, avec un moteur de recherches, sur l'internet au 9 mars 20 I 5, fait apparaitre que de nombreux forums se consacrent au sujet, en particulier ceux de sites dont l'audience féminine est forte : Magicmaman.com, Au féminin.com, Doctissimo.fr, Parolesdemaman.com. II y est évoqué le syndrome d'épuisement des mères qui exercent une activité professionnelle.
} 
HellerT., 2009, « Reconnaissance et gouvernement des salariés. Au-delà du mépris », Questions de communication, 15, pp. 93-107.

Honneth A., 1975, La Lutte pour la reconnaissance, trad. de l'allemand par P. Rusch, Paris, Éd. du Cerf, 2002.

Honneth A., 2006, La Société du mépris. Vers une nouvelle théorie critique, trad. de l'allemand par O. Voirol, P. Rusch, A. Dupeyrix, Paris, Éd. La Découverte.

Jeanneret Y., 2014, Critique de la trivialité. Les Médiations de la communication, enjeu de pouvoir, Le Havre, Éd. Non Standard.

Krieg-Planque A., 2009, La Notion de « formule » en analyse de discours. Cadre théorique et méthodologique, Besançon, Presses universitaires de Franche-Comté.

Krieg-Planque A., 20 I2, Analyser les discours institutionnels, Paris, A. Colin.

Martinet A., 1970, Éléments de linguistique générale, Paris, A. Colin.

Moirand S., 2007, Les Discours de la presse quotidienne. Observer, analyser, comprendre, Paris, Presses universitaires de France.

Mouriquand J., 20 I I, L'Écriture journalistique, Paris, Presses universitaires de France.

Rabatel A., 2010, « Le traitement médiatique des suicides à France Télécom de mai-juin à mi-août 2009 : la lente émergence de la responsabilité du management dans les suicides en lien avec le travail », Studia Universitatis Babes-Bolyai - Studia Philologia, Universitatea BabesBolyai, Lv, pp. 3I-52.

Rabatel A., 20 I I, « La levée progressive du tabou des responsabilités socio-professionnelles dans les suicides en lien avec le travail à France Télécom (fin août-octobre 2009) », Questions de communication, 20, pp. 175-198.

Reinert M., 1983, « Une méthode de classification descendante hiérarchique. Application à l'analyse lexicale par contexte », Les Cahiers de l'analyse des données, VIII, 2, pp. I87- 198.

Reinert M., 2008, « Modes lexicaux stabilisés et analyse statistique de discours 》, IXe Journées internationales d'analyse statistique des données textuelles, pp. 98 I-993.

Ringoot R., 2014, Analyser le discours de presse, Paris, A. Colin.

Rosa H., 2005, Accélération. Une critique sociale du temps, trad. de l'allemand par D. Renault, Paris, Éd. La Découverte, 2010.

Winkin Y., 20 16, « Communication », Encyclopædia Universalis. Accès : http://www.universalis. fr/encyclopedie/communication/.

Zawieja P., 20I5, Le Burn out, Paris, Presses universitaires de France. 